\title{
Design, Synthesis and Pharmacological Evaluation of New Nonsteroidal Anti-Inflammatory Derived from 3-Aminobenzothieno[2,3-d]pyrimidines
}

\author{
Hend Nagah Hafez ${ }^{1,2 *}$, Omar Khalid Al-Duaij ${ }^{1}$, Abdel-Rhman Barakat A. El-Gazzar ${ }^{1,2}$ \\ ${ }^{1}$ Chemistry Department, Faculty of Science, Al-Imam Mohammad Ibn Saud Islamic University (IMSIU), Riyadh, KSA \\ ${ }^{2}$ Photochemistry Department, National Research Centre, Cairo, Egypt \\ Email: "hendnagah2000@yahoo.com
}

Received March 29, 2013; revised May 7, 2013; accepted May 18, 2013

Copyright (C) 2013 Hend Nagah Hafez et al. This is an open access article distributed under the Creative Commons Attribution License, which permits unrestricted use, distribution, and reproduction in any medium, provided the original work is properly cited.

\begin{abstract}
During the last few years, condensed thienopyrimidine derivatives have received considerable attention. The therapeutic importance of thienopyrimidines prompted us to synthesize some of spiro(benzothieno[2,3- $d$ ]pyrimidine-4-one) derivatives. Some of the novel benzothino-pyrimidine derivatives $3 \mathrm{a}, 9 \mathrm{~b}, 10 \mathrm{~b}, 11 \mathrm{a}, 11 \mathrm{~b}$, and 11c showed considerable potent anti-inflammatory and analgesic activity of superior G.I.T. safety profile in experimental rats in comparing to indomethacin and tramadol as reference drugs.
\end{abstract}

Keywords: Spirobenzothienopyrimidine; Triazolopyrimidine; Pyrazolopyrimidine; Analgesic; Anti-Inflammatory; Ulcerogenic Effect

\section{Introduction}

Non-steroidal anti-inflammatory drugs (NSAIDs) are among the most widely used therapeutics, primarily used for the treatment of pain and inflammation in arthritis for significant side effects of gastrointestinal lesions, bleeding, and nephrotoxicity. Therefore, the discovery of new safer anti-inflammatory drugs represents a challenging goal for such a research area, fused pyrimidines continue to attract considerable attention of researchers because of their great practical usefulness, primarily, due to a very wide spectrum of their biological activities. Thienopyrimidines occupy a special position among these compounds. Thienopyrimidine derivatives are characterized by a very broad spectrum of biological activities, such as anticancer [1-4], antiviral [5,6], antimicrobial [7-9], analgesic and anti-inflammatory [10-14] anticonvulsant $[15,16]$, thymidine phosphorylase inhibitors [17], antiHIV [18], and antihistaminic [19]. The present work is an extension of our ongoing efforts towards the synthesis and evaluation of some new substituted thieno[2,3- $d]$ pyrimidine derivatives as analgesic and anti-inflammatory agents.

*Corresponding author.

\section{Results and Discussion}

\subsection{Chemistry}

As a part of our continuing program on the synthesis of anti-inflammatory and analgesic compounds as therapeutics agents, we have earlier reported a series of heterocyclic moieties that have anti-inflammatory and analgesic activities [13,14]. Also, outlines the biological significance of one of the most important heterocyclic thienopyrimidine derivatives. This report deals with the synthesis and the pharmacological evaluation of a series of benzothieno[2,3- $d]$ pyrimidines substituted at C-2 with various groups. The interaction of 5,5-dimethyl cyclohexane-1,3-dione with ethyl-cyanoacetate and sulfur metal in ethanol medium in the presence of diethylamine led to ethyl 2-amino-6,6-dimethyl-4-oxo-4,5,6,7-tetrahydro-1-ben zothiophene-3-carboxylate 1 [13]. The hydrazide compound 2 obtained by refluxing of ethylcarboxylate 1 with hydrazine hydrate in ethanol. The treatment of compound 2-amino-1-benzothiophene-3-carbo hydrazide derivative 2 with 5,5-dimethyl cyclo hexane1,3-dione, cyclohexanone and cyclo pentanone in basic medium (ethanol/pipredine) produced spiro[(3-aminobenzothieno[2,3- $d$ ]pyrimidine-4-one)] derivatives 3a-c (Scheme 1). 

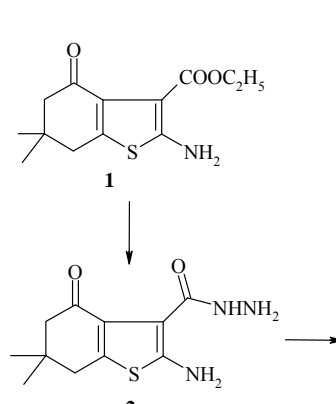

2
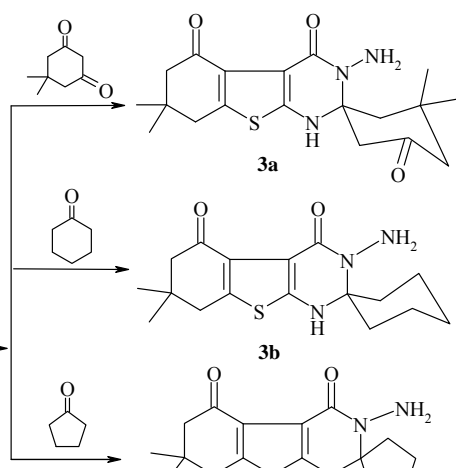

$3 c$

Scheme 1. Synthesis of spiro(3-amino-benzothieno[2,3-d] pyrimidine).

The treatment of ethylcarboxylate (1) with dimethylsulfate and carbon disulfide give (4) which on the treatment with hydrazinehydrate (99\%) afforded 7 , as shown in (Scheme 2). As a model experiment the alkylation of 5 was carried out by the reaction of 1 mol equiv of methyl iodide with the potassium salt (generated in situ by the reaction of 5 with alcoholic potassium hydroxide) generated the new 2-methylthiothieno[2,3- $d]$ pyrimidines 6 . Action of hydrazine hydrate on 3-amino-7,7-dimethyl-2methylthio-3,6,7,8-tetrahydro [1] benzothineno[2,3-d]pyrimidine-4,5-dione (6) in ethanol afforded 2-hydrazino thino[2,3- $d]$ pyrimidine4-one (7).

Structures of these compounds were supported by spectral data such as IR, NMR, mass and elemental analyses. Compound 7 could be considered as a starting material for the synthesis of new polynuclear heterocycles such as pyrazolobenzothinopyrimidine, imidazolobenzothienopyrimidine and triazolobenzothienopyrimidine derivatives. Thus, heating compound 7 with aliphatic acids, namely, formic and acetic acid, resulted in the formation 4-amino-8,9-dihydrobenzothieno[3,2-e][1, $2,4]$ triazolo $[3,4-a]$ pyrimidine-5,6-dione $(\mathbf{8 a}, \mathbf{b})$.

Compound 7 was heated under reflux in glacial acetic acid for $8 \mathrm{hrs}$ with potassium thiocyanate to afford 1,4diamino-8,8-dimethyl-8,9-dihydro[1] benzothieno[3,2-e][1, $2,4]$ triazolo[4,3-a]pyrimidine-5,6-(4H,7H)-dione (8c). When compound 7 was heated under reflux with $\alpha$-haloketones, namely, chloroacetone or 2-bromo aceto-phenone in dry xylene, it yielded the respective 3,4-diamino-2,8,8trimethyl-3,4,8,9-tetrahydro[1]benzothieno[3,2-e]imidazo [1,2-a]pyrimidine-5,6-(3H,7H)-dione (9a). 3,4-Diamino-8,8dimethyl-2-phenyl-3,4,8,9-tetrahydro[1] benzothieno[3,2-e]imidazo[1,2-a] pyrimidine-5,6- $(3 H, 7 H)$-dione $(\mathbf{9 b})$.

Compound 7 reacted with $\beta$-diketone to form $2-(1-$ pyrazolyl)-derivatives. Thus, heating compound 7 with pentane-2,4-dione and/or 3-chloropentane-2,4-dione, yielded 10a,b respectively (Scheme 3 ). On the other hand, fusion of compound $\mathbf{6}$ with morpholine, $N$-methyl piprazine and piprazine in sand bath at $180^{\circ} \mathrm{C}$, yielded 3amino-(morpholinyl/methylpiperazin/ and or piperazinyl) benzothienopyrimidine-4,5-dione 11a-c (Scheme 4).

\subsection{Biological Evaluation}

\subsubsection{Anti-Inflammatory Effect}

The anti-inflammatory activity of fourteen of the newly synthesized compounds 3a, 3b, 3c, 5, 6, 7, 8a, 8c, 9b, 10a, 10b, 11a, 11b, 11c were evaluated by applying carrageenan-induced paw edema bioassay in rats [20] using

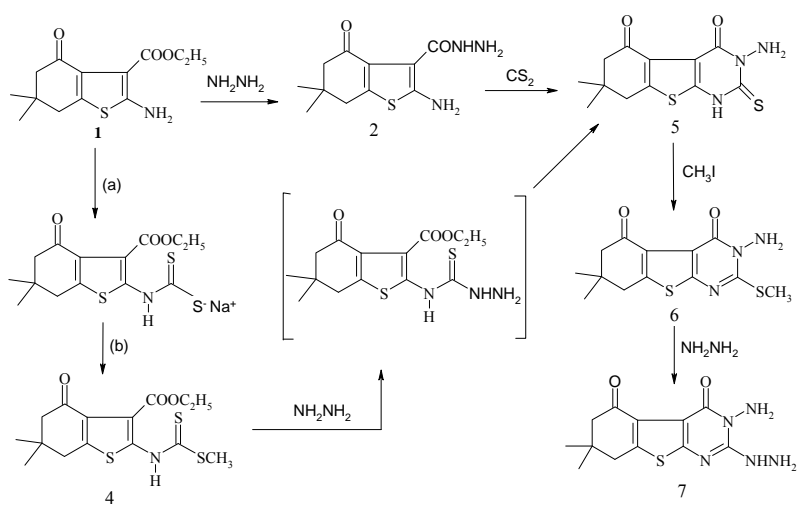

Scheme 2. Synthesis and reaction of benzothinenopyrimidine.

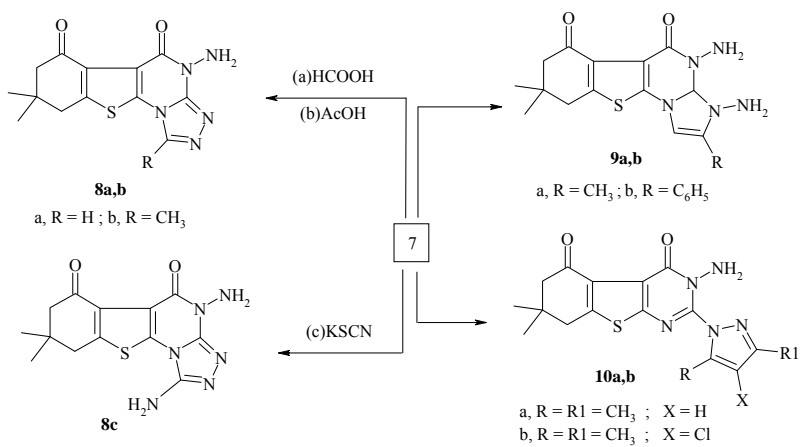

Scheme 3. Synthesis of triazolo and imidazolo pyrimidine.

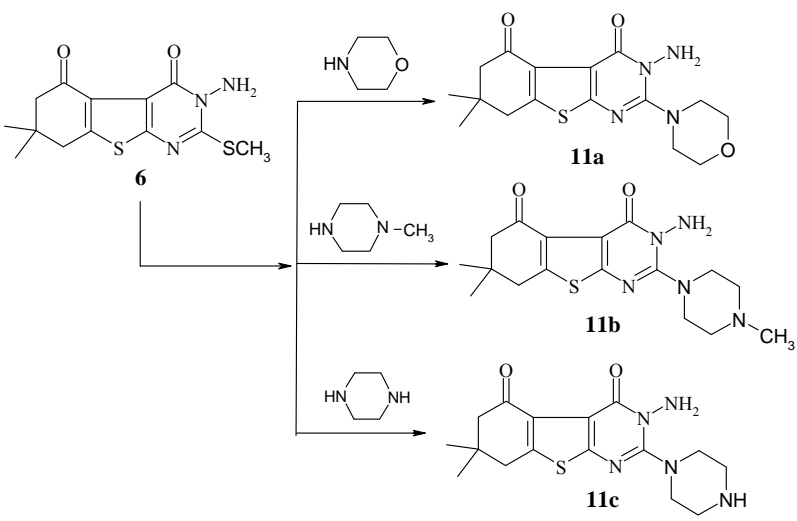

Scheme 4. Synthesis of 3-amino-(2-alkylamino)benzothienopyrimidine-4,5-dione. 
indomethacin as a reference standard. Results were expressed as mean \pm S.E. Difference between vehicle control and treatment groups were tested using one way ANOVA followed by the least significant difference (L.S.D.). Methods of statistical analysis were done according to Armitage et al. [21].

According to Table 1, administration of many of tested compounds 60 min prior to carrageenan injection at dose of $9 \mathrm{mg} / \mathrm{kg} \mathrm{b}$ wt caused significant inhibition of paw edema response. Compounds 3a, 3b, 11a and 11c caused significant decreases in paw edema after 2, 3, $4 \mathrm{~h}$ after drug administration, while $\mathbf{9 b}$ and $\mathbf{1 0 b}$ gave their response after $2 \mathrm{~h}$ of administration and continued to the third hour. Compounds 8c and 11b showed the effect only after $2 \mathrm{~h}$ but compounds 3c, 5, 7 significantly decreased the paw edema after $4 \mathrm{~h}$ post administration.

\subsubsection{Analgesic Activity}

The analgesic activity of the fourteen derivatives was also evaluated by applying Hot plate test [22] using tramadol as a standard reference. Results were expressed as mean \pm S.E. Difference between vehicle control and treatment groups were tested using one way ANOVA followed by the least significant difference (L.S.D.).

Table 1. Anti-inflammatory effect.

\begin{tabular}{|c|c|c|c|c|}
\hline \multirow{2}{*}{$\begin{array}{c}\text { Compd. } \\
\text { No. }\end{array}$} & \multicolumn{4}{|c|}{ Oedema volume } \\
\hline & $1 \mathrm{~h}$ & $2 \mathrm{~h}$ & $3 \mathbf{h}$ & $4 \mathrm{~h}$ \\
\hline Control & $57.3 \pm 6.7$ & $94.4 \pm 8.7$ & $100.3 \pm 3.3$ & $92.3 \pm 3.5$ \\
\hline 3a & $60.4 \pm 7.1$ & $74.1 \pm 5.4^{\mathrm{a}}$ & $80.1 \pm 6.0^{\mathrm{a}}$ & $77.8 \pm 5.5^{\mathrm{a}}$ \\
\hline $3 \mathbf{b}$ & $57.9 \pm 7.2$ & $65.4 \pm 8.8^{\mathrm{a}}$ & $66.4 \pm 6.9^{\mathrm{a}}$ & $68.6 \pm 6.3^{\mathrm{a}}$ \\
\hline $3 c$ & $57.7 \pm 6.3$ & $85.7 \pm 2.8$ & $82.1 \pm 1.3$ & $75.6 \pm 5.1^{\mathrm{a}}$ \\
\hline 5 & $49.9 \pm 2.5$ & $85.7 \pm 2.8$ & $82.1 \pm 1.3$ & $73.4 \pm 2.1^{\mathrm{a}}$ \\
\hline 6 & $61.7 \pm 5.0$ & $79.3 \pm 3.0$ & $85.1 \pm 3.6$ & $78.6 \pm 8.3$ \\
\hline 7 & $49.9 \pm 2.5$ & $88.7 \pm 6.1$ & $83.5 \pm 3.7$ & $73.4 \pm 2.1^{\mathrm{a}}$ \\
\hline $8 \mathbf{a}$ & $54.9 \pm 6.2$ & $66.7 \pm 6.9^{\mathrm{a}}$ & $77.2 \pm 6.6^{\mathrm{a}}$ & $79.6 \pm 4.9$ \\
\hline $8 c$ & $85.4 \pm 3.1$ & $74.6 \pm 7.5^{\mathrm{a}}$ & $88.0 \pm 7.2$ & $89.9 \pm 2.1$ \\
\hline $9 b$ & $49.4 \pm 7.1$ & $71.8 \pm 6.7^{\mathrm{a}}$ & $76.4 \pm 4.8^{\mathrm{a}}$ & $82.2 \pm 5.2$ \\
\hline $10 a$ & $67.5 \pm 7.7$ & $83.4 \pm 5.2$ & $88.6 \pm 5.3$ & $94.7 \pm 7.4$ \\
\hline $10 b$ & $44.2 \pm 5.1$ & $59.6 \pm 4.7^{\mathrm{a}}$ & $67.8 \pm 3.3^{\mathrm{a}}$ & $81.9 \pm 3.2$ \\
\hline $11 a$ & $61.0 \pm 6.6$ & $66.6 \pm 5.9^{\mathrm{a}}$ & $68.6 \pm 7.0^{\mathrm{a}}$ & $72.4 \pm 7.4^{*}$ \\
\hline $11 b$ & $66.4 \pm 7.5$ & $78.2 \pm 3.5^{\mathrm{a}}$ & $81.3 \pm 3.3$ & $87.1 \pm 2.1$ \\
\hline 11c & $56.2 \pm 9.9$ & $65.1 \pm 7.5^{\mathrm{a}}$ & $55.9 \pm 10.6^{\mathrm{a}}$ & $54.7 \pm 7.2^{\mathrm{a}}$ \\
\hline Indomethacine & $49.8 \pm 5.3$ & $42.9 \pm 5.1^{\mathrm{a}}$ & $45.9 \pm 4.6^{\mathrm{a}}$ & $46.9 \pm 5.8^{\mathrm{a}}$ \\
\hline
\end{tabular}

${ }^{\mathrm{a} P}<0.05$ : Statistically significant from the control using one way ANOVA (Two-sided Dunnett as Post Hoc test).
Methods of statistical analysis were done according to Armitage et al. [21].

According to Table 2, compounds 3a, 8a, 10a, 11a and 11c showed significant analgesic activity higher than that obtained by Tramadol $1 \mathrm{~h}$ and $2 \mathrm{~h}$ post administration. Compounds 8c, 10b and 11b exhibited significant analgesic activity higher than or slightly equipotent to Tramadol only after $2 \mathrm{~h}$ of administration. Compounds $3 \mathbf{b}$ and 5 exhibited the analgesic effect after $1 \mathrm{~h}$ of administration only. Compounds 3c, 6, 7 and $\mathbf{9 b}$ have no analgesic activity Thus, it can be concluded that, compounds 3a, b, 5, 8a, c, 10a, b, 11a-c have significant analgesic activity and compound 11a is the most potent compound.

\subsubsection{Ulcerogenic Effect}

The ulcerogenic effect of the most active anti-inflammatory and analgesic derivatives 3a, b, 8a, 10a, 11a, c was evaluated [23]. According to Table 3, it has been found that, compounds 3a, b, 8a, 11c have very little ulcerogenic effect with in comparison to indomethacin. Interestingly, compound 11a exhibited no ulcerogenic effect in all of the experimental animals. On the other hand compound 10a resulted in ulcer lesions in many of

Table 2. Analgesic effect.

\begin{tabular}{|c|c|c|c|}
\hline \multirow{2}{*}{ Compounds } & \multicolumn{3}{|c|}{ Percent analgesic activity } \\
\hline & Basal & $1 \mathrm{~h}$ & $2 \mathrm{~h}$ \\
\hline Control & $12.2 \pm 0.63$ & $12.1 \pm 0.83$ & $12.2 \pm 1.18$ \\
\hline 3a & $10.9 \pm 1.03$ & $19.8 \pm 1.59^{\mathrm{a}}$ & $21.6 \pm 1.54^{\mathrm{a}}$ \\
\hline $3 \mathbf{b}$ & $9.2 \pm 0.83$ & $10.9 \pm 0.56$ & $15.3 \pm 1.17$ \\
\hline $3 c$ & $11.8 \pm 0.82$ & $13.5 \pm 0.75$ & $11.5 \pm 0.85$ \\
\hline 5 & $9.4 \pm 0.51$ & $17.0 \pm 0.43^{\mathrm{a}}$ & $13.6 \pm 1.14$ \\
\hline 6 & $12.0 \pm 0.92$ & $14.6 \pm 1.24$ & $15.6 \pm 1.02$ \\
\hline 7 & $9.2 \pm 0.83$ & $10.9 \pm 0.56$ & $15.3 \pm 1.17$ \\
\hline $8 \mathbf{8}$ & $11.0 \pm 0.91$ & $19.1 \pm 1.46^{\mathrm{a}}$ & $19.2 \pm 1.00^{\mathrm{a}}$ \\
\hline $8 c$ & $13.3 \pm 1.33$ & $16.9 \pm 1.18$ & $18.1 \pm 1.36^{\mathrm{a}}$ \\
\hline $9 b$ & $8.9 \pm 0.73$ & $13.0 \pm 1.03$ & $16.1 \pm 1.20$ \\
\hline $10 a$ & $11.8 \pm 0.82$ & $13.5 \pm 0.75$ & $11.5 \pm 0.85$ \\
\hline $10 \mathrm{~b}$ & $10.3 \pm 1.03$ & $14.6 \pm 0.93$ & $18.4 \pm 1.47$ \\
\hline $11 a$ & $15.1 \pm 1.34$ & $24.9 \pm 1.38^{\mathrm{a}}$ & $19.4 \pm 0.61^{\mathrm{a}}$ \\
\hline $11 b$ & $12.9 \pm 0.95$ & $15.0 \pm 1.02$ & $20.5 \pm 1.19^{\mathrm{a}}$ \\
\hline 11c & $12.6 \pm 1.13$ & $21.0 \pm 2.47^{\mathrm{a}}$ & $18.4 \pm 0.69^{\mathrm{a}}$ \\
\hline Tramadol & $13.1 \pm 0.78$ & $17.6 \pm 0.32^{\mathrm{a}}$ & $18.2 \pm 0.28^{\mathrm{a}}$ \\
\hline
\end{tabular}

Values represent the mean \pm S.E. of six animals for each groups. ${ }^{a} \mathrm{P}<0.05$ : Statistically significant from Control (Dunnett's test). 
Table 3. Ulcerogenic effect.

\begin{tabular}{cccc}
\hline \multirow{2}{*}{ Compounds } & \multicolumn{3}{c}{ Ulcer index } \\
\cline { 2 - 4 } & No. of ulcer & Severity of ulcer & $\begin{array}{c}\text { No. of rats } \\
\text { with ulcer/5 }\end{array}$ \\
\hline Control & $8.2 \pm 0.86$ & $19.4 \pm 2.20$ & 5 \\
3a & $0.4 \pm 0.40^{\mathrm{a}}$ & $0.6 \pm 0.60^{\mathrm{a}}$ & 1 \\
3b & $1.6 \pm 1.36^{\mathrm{a}}$ & $2.0 \pm 1.76^{\mathrm{a}}$ & 2 \\
8a & $2.6 \pm 1.66^{\mathrm{a}}$ & $3.2 \pm 1.96^{\mathrm{a}}$ & 2 \\
10a & $7.0 \pm 1.64$ & $10.4 \pm 2.86$ & 5 \\
11a & $0.0 \pm 0.00^{\mathrm{a}}$ & $0.0 \pm 0.00^{\mathrm{a}}$ & 0 \\
11c & $0.4 \pm 0.40^{\mathrm{a}}$ & $0.4 \pm 0.40^{\mathrm{a}}$ & 1 \\
Indomethacine & $5.8 \pm 1.77$ & $9.8 \pm 3.23^{\mathrm{a}}$ & 5 \\
\hline
\end{tabular}

Values represent the mean \pm S.E. of five animals for each group. ${ }^{\text {a }} \mathrm{P}<0.05$ : Statistically significant from ethanol treated rats (Kruskal Wallis, followed by Mann Whitney test).

the experimental rats. Therefore, the potential medicinal value of these compounds as anti-inflammatory and analgesic agents, that they have better safety margin than indomethacin on gastric mucosa.

\section{Conclusion}

Different fourteen compounds were evaluated as antiinflammatory and analgesic agents in experimental animals. It has been found that the compounds $\mathbf{3 a}, \mathbf{3 b}, \mathbf{8 a}$, 10a, 11a, 11c exhibited the dual pharmacological activities with superior gastrointestinal safety profile when compared to indomethacin except 10a which resulted in ulcer lesions in many of the experimental rats. Surprisingly, compound 11a exhibited no ulcerogenic effect in all of the experimental animals. Thus, it can be concluded that spirobenzothienopyrimidine moiety, phenylpyrazolo-thinopyrimidine, morphonyl and piperazinylthinopyrimidine ring systems are important for both anti-inflammatory and analgesic activity of potent safety margin profiles towards gastrointestinal tract.

\section{Experimental}

\subsection{Chemistry}

Melting points were determined on the Electrothermal 9100 melting point apparatus (Electrothermal, UK) and were uncorrected. The IR spectra $(\mathrm{KBr})$ were recorded on an FT-IR NEXCES spectrophotometer. The ${ }^{1} \mathrm{H}$ NMR spectra were measured with a Jeol ECA $500 \mathrm{MHz}$ (Japan) in DMSO- $d_{6}$ or $\mathrm{CDCl}_{3}$ and chemical shifts were recorded in $\delta \mathrm{ppm}$ relative to TMS. Mass spectra (EI) were run at $70 \mathrm{eV}$ with a Finnigan SSQ 7000 spectrometer. The purity of the compounds was checked on Aluminium plates coated with silica gel (Merck). The pharmacological eva- luations of the products were carried out in Pharmacological Unit Pharmacology Department (NRC, Cairo, Egypt).

Synthesis of ethyl 2-amino-6,6-dimethyl-4-oxo-4,5, 6,7-tetrahydro-1-benzothiophene-3-carboxylate (1). A mixture of 5,5-dimethyl cyclohexane-1,3-dione $(10 \mathrm{~m}$ $\mathrm{mol})$, ethylcyano-acetate $(10 \mathrm{~m} \mathrm{~mol})$, sulfur $(10 \mathrm{~m} \mathrm{~mol})$ and diethylamine $(15 \mathrm{~m} \mathrm{~mol})$ was heated under stirring in absolute ethanol for $4 \mathrm{~h}$, then leave the mixture for $24 \mathrm{~h}$ at $0^{\circ} \mathrm{C}$. The formed solid was collected by filtration, washed with ethanol $(20 \mathrm{~mL})$, dried and crystallized from absolute ethanol, as white crystals in a $72 \%$ yield, m.p. $168^{\circ} \mathrm{C}-170^{\circ} \mathrm{C}$; IR $\left(\mathrm{cm}^{-1}\right.$, v); 3420 (br, NH), 3042, 2917 (CH alkyl), 1720, $1706(2 \mathrm{C}=\mathrm{O}) ;{ }^{1} \mathrm{H}$ NMR (DMSO- $\left.d_{6}\right): \delta$ $0.82\left(\mathrm{~s}, 3 \mathrm{H}, \mathrm{CH}_{3}\right), 0.93\left(\mathrm{~s}, 3 \mathrm{H}, \mathrm{CH}_{3}\right), 2.12(\mathrm{~d}, J=17.0 \mathrm{~Hz}$, $\left.2 \mathrm{H}, \mathrm{CH}_{2}\right), 2.33\left(\mathrm{~d}, J=17.0 \mathrm{~Hz}, 2 \mathrm{H}, \mathrm{CH}_{2}\right), 1.58(\mathrm{t}, 3 \mathrm{H}$, $\left.\mathrm{CH}_{3}\right) 4.08$ (q, 2H, $\mathrm{CH}_{2}$ ), 7.73 (br, $2 \mathrm{H}, \mathrm{NH}_{2}, \mathrm{D}_{2} \mathrm{O}$ exchangeable); Its MS (m/z) $267\left(\mathrm{M}^{+}, 67 \%\right) ; \mathrm{C}_{13} \mathrm{H}_{17} \mathrm{NO}_{3} \mathrm{~S}$ (267.3); Requires (Found): C, 58.40 (58.38); H, 6.41 (6.37); N, 5.23 (5.20).

Synthesis of 2-amino-6,6-dimethyl-4-oxo-4,5,6,7tetrahydro-1-benzothiophene-3-carbohydrazide (2). A suspension of dry compound $\mathbf{1}(10 \mathrm{~m} \mathrm{~mol})$ in hydrazine hydrate $(80 \%)(5 \mathrm{~mL})$ was stirred under gentle reflux. The insoluble solid dissolved within 10 min with evolution of hydrogen sulfide to form a clear solution. After $0.5 \mathrm{~h}$. when the solid product started separating out, heating was continued for $8 \mathrm{~h}$. The reaction mixture was then allowed to cool to room temperature. The solid was filtered, washed with ethanol, dried and crystallized from dioxane; as yellow crystals in a $85 \%$ yield, m.p. $210^{\circ} \mathrm{C}$ $213^{\circ} \mathrm{C}$; IR $\left(\mathrm{cm}^{-1}, v\right): 3400(\mathrm{br}, \mathrm{NH}), 1715,1690(2 \mathrm{C}=\mathrm{O})$; ${ }^{1} \mathrm{H}$ NMR (DMSO- $\left.d_{6}\right): \delta 0.95\left(\mathrm{~s}, 3 \mathrm{H}, \mathrm{CH}_{3}\right), 1.00(\mathrm{~s}, 3 \mathrm{H}$, $\left.\mathrm{CH}_{3}\right), 1.96$ (d, $\left.J=16.9 \mathrm{~Hz}, 2 \mathrm{H}, \mathrm{CH}_{2}\right), 2.14$ (d, $J=17.0$ $\mathrm{Hz}, 2 \mathrm{H}, \mathrm{CH}_{2}$ ), 4.35 (br, $\left.2 \mathrm{H}, \mathrm{NH}_{2}\right), 7.63$ (br, $2 \mathrm{H}, \mathrm{NH}_{2}$ ), $8.93(\mathrm{br}, 1 \mathrm{H}, \mathrm{NH})\left(\mathrm{NH}, 2 \mathrm{NH}_{2}, \mathrm{D}_{2} \mathrm{O}\right.$ exchangeable); Its MS (m/z), $253\left(\mathrm{M}^{+}, 29 \%\right) ; \mathrm{C}_{11} \mathrm{H}_{15} \mathrm{~N}_{3} \mathrm{O}_{2} \mathrm{~S}$ (253.3); Requires (Found): C, 52.15 (52.13); H, 5.96 (5.92); N, 16.58 (16.53).

Synthesis of Spiro(benzothieno[2,3-d]pyrimidine-4one) (3a-c). General procedure: A mixture of compound $2(10 \mathrm{~m} \mathrm{~mol})$ with 5,5-dimethyl cyclohexane-1,3dione/or cyclohexanone/and or cyclopentanone was refluxed in basic medium formed from (ethanol/ pipredine). The solid that separated upon cooling was filtered off and crystallized from appropriate solvent to produced (3a-c).

Synthesis of 3-amino-5',5',7,7-tetramethyl-7,8-dihydro-1H,3' $H$-spiro[1-benzothieno[2,3-d]pyrimidine-2, 1'-cyclohexane]-3',4,5(3H,6H)-trione (3a). A mixture of compound $2(10 \mathrm{~m} \mathrm{~mol})$ with 5,5-dimethyl cyclohexane-1,3-dione $(10 \mathrm{~m} \mathrm{~mol})$ was heated under reflux in (ethanol/ pipredine) for $8 \mathrm{~h}$. The solid that separated upon cooling was filtered off and crystallized from dimethylformamide, as orange powder, in a 70\% yield; m.p. 
$250^{\circ} \mathrm{C}-252^{\circ} \mathrm{C}$; IR $\left(\mathrm{cm}^{-1}, v\right): 3300$ (br, NH's), 1718, 1710, $1686(3 \mathrm{C}=\mathrm{O}) ;{ }^{1} \mathrm{H}$ NMR (DMSO- $\left.d_{6}\right): \delta, 0.84\left(\mathrm{~s}, 3 \mathrm{H}, \mathrm{CH}_{3}\right)$, $0.87\left(\mathrm{~s}, 3 \mathrm{H}, \mathrm{CH}_{3}\right), 0.96\left(\mathrm{~s}, 3 \mathrm{H}, \mathrm{CH}_{3}\right), 1.20\left(\mathrm{~s}, 3 \mathrm{H}, \mathrm{CH}_{3}\right)$, $1.43\left(\mathrm{~d}, 2 \mathrm{H}, \mathrm{CH}_{2}\right), 1.78$ (d, $\left.2 \mathrm{H}, \mathrm{CH}_{2}\right), 1.97\left(\mathrm{~d}, 2 \mathrm{H}, \mathrm{CH}_{2}\right)$, $2.28\left(\mathrm{~d}, 2 \mathrm{H}, \mathrm{CH}_{2}\right) 2.33\left(\mathrm{~d}, 2 \mathrm{H}, \mathrm{CH}_{2}\right), 7.90(\mathrm{br}, \mathrm{NH}), 8.83$ (br, $1 \mathrm{H}, \mathrm{NH}) ;\left(2 \mathrm{NH}, \mathrm{D}_{2} \mathrm{O}\right.$ exchangeable); ${ }^{13} \mathrm{C}$ NMR (DMSO- $\left.d_{6}\right): \delta, 190.3,187.1$ (2CO), 164.6 (CO amide), $141.6,139.5,124.7,114.2$ (thiophene carbons), 72.54 (spiro head), 51.42, 50.09, 44.23, 43.29, $41.09\left(5 \mathrm{CH}_{2}\right)$, $38.79\left(\mathrm{C}\left(\mathrm{CH}_{3}\right)_{2}\right), 28.98,28.24,27.57,26.32\left(4 \mathrm{CH}_{3}\right)$; Its MS (m/z), $375\left(\mathrm{M}^{+}, 68 \%\right) ; \mathrm{C}_{19} \mathrm{H}_{25} \mathrm{~N}_{3} \mathrm{O}_{3} \mathrm{~S}$ (375.4); Requires (Found): C, 60.77 (60.73); H, 6.71 (6.69); N, 11.19 (11.15).

Synthesis of 3-amino-7,7-dimethyl-7,8-dihydro-1Hspiro[1-benzothieno[2,3-d]pyrimidine-2,1'-cyclohex ane]-4,5-(3H,6H)-dione (3b). It was obtained from the reaction of $2(10 \mathrm{~m} \mathrm{~mol})$ with cyclohexanone $(10 \mathrm{~m} \mathrm{~mol})$ as yellow powder crystallized from dimethylformamide, in a $65 \%$ yield, m.p. $260^{\circ} \mathrm{C}-263^{\circ} \mathrm{C}$; IR $\left(\mathrm{cm}^{-1}, v\right): 3430$ (br, NH), 1711, $1690(2 \mathrm{C}=\mathrm{O})$; ${ }^{1} \mathrm{H}$ NMR (DMSO- $\left.d_{6}\right): \delta$ 0.89 (s, 3H, $\left.\mathrm{CH}_{3}\right), 0.95$ (s, 3H, $\left.\mathrm{CH}_{3}\right), 2.12\left(\mathrm{~d}, 2 \mathrm{H}, \mathrm{CH}_{2}\right)$, $2.33\left(\mathrm{~d}, 2 \mathrm{H}, \mathrm{CH}_{2}\right), 2.53-3.05\left(\mathrm{~m}, 10 \mathrm{H}, 5 \mathrm{CH}_{2}\right), 8.00$ (br, $1 \mathrm{H}, \mathrm{NH}), 9.07$ (br, 1H, NH) (2NH, $\mathrm{D}_{2} \mathrm{O}$ exchangeable); ${ }^{13} \mathrm{C}$ NMR (DMSO- $\left.d_{6}\right): \delta, 189.2$ (CO), 165.8 (CO amide), $141.8,139.2,123.9,113.8$ (thiophene carbons), 72.09

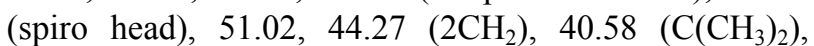
40.29 - 37.89 (cyclohexane carbons), 29.03, 28.19, $\left(2 \mathrm{CH}_{3}\right)$; Its MS (m/z), $333\left(\mathrm{M}^{+}, 57 \%\right) ; \mathrm{C}_{17} \mathrm{H}_{23} \mathrm{~N}_{3} \mathrm{O}_{2} \mathrm{~S}$ (333.4); Requires (Found): C, 61.23 (61.20); H, 6.95 (6.91); N, 12.60 (12.57).

Synthesis of 3-amino-7,7-dimethyl-7,8-dihydro-1Hspiro[1-benzothieno[2,3-d]pyrimidine-2,1'-cyclopentane]-4,5-(3H,6H)-dione (3c). It was obtained from the reaction of $2(10 \mathrm{~m} \mathrm{~mol})$ with cyclopentanone $(10 \mathrm{~m} \mathrm{~mol})$ as white powder crystallized from dimethylformamide in a $67 \%$ yield; m.p. $235^{\circ} \mathrm{C}-237^{\circ} \mathrm{C}$; IR $\left(\mathrm{cm}^{-1}\right.$, v); $3285(\mathrm{br}$, $\mathrm{NH}), 1721,1673(2 \mathrm{C}=\mathrm{O}) ;{ }^{1} \mathrm{H}$ NMR (DMSO- $\left.d_{6}\right): \delta 0.91$ (s, $\left.3 \mathrm{H}, \mathrm{CH}_{3}\right), 0.97\left(\mathrm{~s}, 3 \mathrm{H}, \mathrm{CH}_{3}\right), 2.17\left(\mathrm{~m}, 4 \mathrm{H}, 2 \mathrm{CH}_{2}\right)$, $2.43\left(\mathrm{~d}, 2 \mathrm{H}, \mathrm{CH}_{2}\right), 2.71-2.75\left(\mathrm{~m}, 2 \mathrm{H}, \mathrm{CH}_{2}\right), 2.84-2.95$ (m, 2H, $\mathrm{CH}_{2}$ ), $2.99-3.33$ (m, $2 \mathrm{H}, \mathrm{CH}_{2}$ ); Its $\mathrm{MS}(\mathrm{m} / \mathrm{z})$, $319\left(\mathrm{M}^{+}, 36 \%\right) ; \mathrm{C}_{16} \mathrm{H}_{21} \mathrm{~N}_{3} \mathrm{O}_{2} \mathrm{~S}$ (319.4); Requires (Found): C, 60.16 (60.12); H, 6.62 (6.59); N, 13.15 (13.11).

Synthesis of ethyl 6,6-dimethyl-2-\{I(methylthio) carbon thioyl]amino\}-4-oxo-4,5,6,7-tetrahydro-1-benzothiophene-3-carboxylate (4). To a vigorously stirred solution of ethyl 2-amino-benzothiophene-3-carboxylate (1) $(20 \mathrm{~m} \mathrm{~mol})$ in dimethylsulfoxide $(10 \mathrm{~mL})$ at room temperature, carbon disulfide $(25 \mathrm{~m} \mathrm{~mol})$ and aqueous sodium hydroxide $(1.2 \mathrm{~mL}, 20 \mathrm{~mol}$ solution) were added simultaneously over $0.5 \mathrm{~h}$. the stirring was continued for further $30 \mathrm{~min}$. Dimethylsulfate $(20 \mathrm{~m} \mathrm{~mol})$ was added drop wise to the reaction mixture with stirring at $5^{\circ} \mathrm{C}$ $10^{\circ} \mathrm{C}$, it was further stirred for $2 \mathrm{~h}$. and poured into ice-water, the solid obtained was filtered, dried and crys- tallized from ethanol as yellow powder; in $87 \%$ yield, m.p. $120^{\circ} \mathrm{C}-122^{\circ} \mathrm{C}$; IR $\left(\mathrm{cm}^{-1}\right.$, v): $3260(\mathrm{br}, \mathrm{NH}), 1727$, $1668(2 \mathrm{C}=\mathrm{O}) ;{ }^{1} \mathrm{H}$ NMR (DMSO- $\left.d_{6}\right): \delta 1.00\left(\mathrm{~s}, 3 \mathrm{H}, \mathrm{CH}_{3}\right)$, $1.04\left(\mathrm{~s}, 3 \mathrm{H}, \mathrm{CH}_{3}\right), 1.89\left(\mathrm{t}, 3 \mathrm{H}, \mathrm{CH}_{3}\right) 2.19\left(\mathrm{~m}, 4 \mathrm{H}, 2 \mathrm{CH}_{2}\right)$, 3.89 (q, 2H, $\left.\mathrm{OCH}_{2}\right), 8.70(\mathrm{br}, \mathrm{NH})$; Its $\mathrm{MS}(\mathrm{m} / \mathrm{z}), 357$ $\left(\mathrm{M}^{+}, 21 \%\right) ; \mathrm{C}_{15} \mathrm{H}_{19} \mathrm{NO}_{3} \mathrm{~S}_{3}$ (357.4); Requires (Found): $\mathrm{C}$, 50.39 (50.36); H, 5.35 (5.33); N, 3.91 (3.88).

Synthesis of 3-amino-7,7-dimethyl-2-thioxo-1,2,3, 6,7,8-hexahydro[1]benzothieno[2,3-d]pyrimidine-4,5dione (5). To a solution of $4(10 \mathrm{~m} \mathrm{~mol})$ in ethanol 30 $\mathrm{mL}$ was treated with hydrazine hydrate $(10 \mathrm{~m} \mathrm{~mol}, 99 \%)$ and refluxed on a water bath until the methyl-mercaptan evolution ceases $(8 \mathrm{~h})$. After cooling, the solid obtained was filtered, dried and recrystallized from ethanol/acetone mixture as brown crystals; in $85 \%$ yield, m.p. $180^{\circ} \mathrm{C}-183^{\circ} \mathrm{C}$; IR $\left(\mathrm{cm}^{-1}, v\right): 3410$ (brs, NH's), 1714 , $1666(2 \mathrm{C}=\mathrm{O}) ;{ }^{1} \mathrm{H}$ NMR (DMSO- $\left.d_{6}\right): \delta 0.99\left(\mathrm{~s}, 3 \mathrm{H}, \mathrm{CH}_{3}\right)$, $1.02\left(\mathrm{~s}, 3 \mathrm{H}, \mathrm{CH}_{3}\right), 2.13\left(\mathrm{~m}, 4 \mathrm{H}, 2 \mathrm{CH}_{2}\right), 8.87,9.53$ (brs, $2 \mathrm{NH}$ 's); ${ }^{13} \mathrm{C}$ NMR (DMSO- $\left.d_{6}\right): \delta, 188.3(\mathrm{C}=\mathrm{O}), 174.4$ (CS), 165.4 (CO amide), 140.2, 139.1, 124.5, 114.3 (thio-

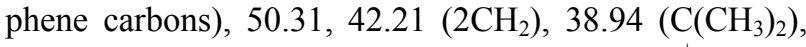
29.34, $27.42\left(2 \mathrm{CH}_{3}\right)$; Its $\mathrm{MS}(\mathrm{m} / \mathrm{z}), 295\left(\mathrm{M}^{+}, 52 \%\right)$; $\mathrm{C}_{12} \mathrm{H}_{13} \mathrm{~N}_{3} \mathrm{O}_{2} \mathrm{~S}_{2}$ (295.3); Requires (Found): C, 48.79 (48.76); H, 4.43 (4.40); N, 14.22 (14.20).

The second way for preparation. To a warmed ethanolic sodium hydroxide solution $(0.40 \mathrm{~g}$ in $50 \mathrm{~mL}$ ethanol), compound $2(10 \mathrm{~m} \mathrm{~mol})$, and carbon disulfide (excess $5 \mathrm{~mL}$ ) were added. The mixture was heated under reflux for $15 \mathrm{~h}$. The reaction mixture was allowed to cool to $0^{\circ} \mathrm{C}$, the deposited precipitate was filtered off, washed by water $(20 \mathrm{~mL})$, dried, and crystallized from ethanol/acetone mixture as brown crystals; in $68 \%$ yield, m.p. $180^{\circ} \mathrm{C}-183^{\circ} \mathrm{C}$.

Synthesis of 3-amino-7,7-dimethyl-2-(methylthio)3,6,7,8-tetrahydro[1]benzothieno[2,3- $d$ ] pyrimidine-4, 5-dione (6). To a warmed ethanolic $\mathrm{KOH}$ solution prepared by dissolving $(10 \mathrm{~m} \mathrm{~mol})$ of $\mathrm{KOH}$ in $50 \mathrm{~mL}$ (ethanol) was added each of compound $5(10 \mathrm{~m} \mathrm{~mol})$, the heating was continued for $30 \mathrm{~min}$ and the mixture was allowed to cool to room temperature, and the proper methyliodide $(12 \mathrm{~m} \mathrm{~mol})$ was added. The mixture was stirred under reflux for $5 \mathrm{~h}$, then cool to room temperature, poured into cold water $(100 \mathrm{~mL})$. The solid product precipitated was filtered off washed with $100 \mathrm{~mL}$ water. The product was dried and crystallized from dioxane as a yellow powder, in yield $85 \%$, m.p. $205^{\circ} \mathrm{C}-207^{\circ} \mathrm{C}$, IR $\left(\mathrm{cm}^{-1}\right.$, v): 3415 (brs, NH's), 1718, $1668(2 \mathrm{C}=\mathrm{O}) ;{ }^{1} \mathrm{H}$ NMR (DMSO- $\left.d_{6}\right): \delta 0.98\left(\mathrm{~s}, 3 \mathrm{H}, \mathrm{CH}_{3}\right), 1.01\left(\mathrm{~s}, 3 \mathrm{H}, \mathrm{CH}_{3}\right)$, $2.11\left(\mathrm{~m}, 4 \mathrm{H}, 2 \mathrm{CH}_{2}\right), 2.34$ (s, 3H, $\left.\mathrm{SCH}_{3}\right), 8.85,9.52$ (brs, 2NH's); Its MS (m/z), $309\left(\mathrm{M}^{+}, 38 \%\right) ; \mathrm{C}_{13} \mathrm{H}_{15} \mathrm{~N}_{3} \mathrm{O}_{2} \mathrm{~S}_{2}$ (309.4); Requires (Found): C, 50.46 (50.44); H, 4.88 (4.85); N, 13.58 (13.54).

Synthesis of 3-amino-2-hydrazino-7,7-dimethyl-3,6, 7,8-tetrahydro[1]benzothieno[2,3-d]pyrimidine-4,5- 
dione (7). A suspension of compound 6 (10 m mol) in hydrazine hydrate $(99 \%, 20 \mathrm{ml})$ was stirred under reflux for $10 \mathrm{~h}$. The reaction mixture was allowed to cool to room temperature. The solid precipitated was filtered off, washed with ethanol, dried and crystallized from dimethylformamide to produce 7 as white powder in $90 \%$ yield; m.p. $278^{\circ} \mathrm{C}-280^{\circ} \mathrm{C}$, IR ( $\left.\mathrm{cm}^{-1}, v\right): 3455$ (brs, NH's), $1721,1669(2 \mathrm{C}=\mathrm{O}) ;{ }^{1} \mathrm{H} \mathrm{NMR}\left(\mathrm{DMSO}-d_{6}\right) \mathrm{ppm}: \delta 1.02(\mathrm{~s}$, $\left.3 \mathrm{H}, \mathrm{CH}_{3}\right), 1.13\left(\mathrm{~s}, 3 \mathrm{H}, \mathrm{CH}_{3}\right), 2.17\left(\mathrm{~m}, 4 \mathrm{H}, 2 \mathrm{CH}_{2}\right), 8.95$, 9.52 ( brs, NH's); ${ }^{13} \mathrm{C}$ NMR (DMSO- $\left.d_{6}\right): \delta, 189.4(\mathrm{C}=\mathrm{O})$, 165.7 (CO amide), 161.4 (C-2, pyrimidine), 142.1, 139.3, 125.1, 115.6 (thiophene carbons), 51.32, $43.27\left(2 \mathrm{CH}_{2}\right)$, $39.71\left(\mathrm{C}\left(\mathrm{CH}_{3}\right)_{2}\right), 28.98,27.57\left(2 \mathrm{CH}_{3}\right)$; Its $\mathrm{MS}(\mathrm{m} / \mathrm{z}), 293$ $\left(\mathrm{M}^{+}, 52 \%\right) ; \mathrm{C}_{12} \mathrm{H}_{15} \mathrm{~N}_{5} \mathrm{O}_{2} \mathrm{~S}$ (293.3); Requires (Found): $\mathrm{C}$, 49.13 (49.10); H, 5.15 (5.13); N, 23.87 (23.84).

Synthesis of 4-amino-8,8-dimethyl-8,9-dihydro[1] benzothieno[3,2-e][1,2,4] triazolo[4,3-a]pyrimidine-5, 6-(4H,7H)-dione (8a). A mixture of $7(10 \mathrm{~m} \mathrm{~mol})$ and formic acid $(10 \mathrm{~mL})$ and $2 \mathrm{~mL}$ of concentrated hydrochloric acid was heated under reflux for $8 \mathrm{~h}$. The reaction mixture was allowed to cool to room temperature and was poured into water $(100 \mathrm{~mL})$. The solid formed was collected by filtration, washed with ethanol $(20 \mathrm{~mL})$, dried and crystallized from DMF as an orange powder in $80 \%$ yield; m.p. $255^{\circ} \mathrm{C}-257^{\circ} \mathrm{C}$, IR $\left(\mathrm{cm}^{-1}, v\right): 3423(\mathrm{br}$, $\mathrm{NH}), 1712,1665(2 \mathrm{C}=\mathrm{O}) ;{ }^{1} \mathrm{H}$ NMR (DMSO- $\left.d_{6}\right): \delta 0.99$ $\left(\mathrm{s}, 3 \mathrm{H}, \mathrm{CH}_{3}\right), 1.02\left(\mathrm{~s}, 3 \mathrm{H}, \mathrm{CH}_{3}\right), 2.12\left(\mathrm{~m}, 4 \mathrm{H}, 2 \mathrm{CH}_{2}\right)$, 6.31 (s, 1H, triazol proton), 9.52 ( br, NH); Its $\mathrm{MS}(\mathrm{m} / \mathrm{z})$, $303\left(\mathrm{M}^{+}, 44 \%\right) ; \mathrm{C}_{13} \mathrm{H}_{13} \mathrm{~N}_{5} \mathrm{O}_{2} \mathrm{~S}$ (303.3); Requires (Found): C, 51.47 (51.44); H, 4.31 (4.29); N, 23.09 (23.07).

Synthesis of 4-amino-1,8,8-trimethyl-8,9-dihydro[1] benzothieno[3,2-e][1,2,4] triazolo[4,3-a]pyrimidine-5, 6-(4H,7H)-dione (8b). A mixture of $7(10 \mathrm{~m} \mathrm{~mol})$ and glacial acetic acid $(50 \mathrm{~mL})$ was stirred under reflux for $10 \mathrm{~h}$ (TLC). The reaction mixture was allowed to cool to room temperature an was then poured into water (100 $\mathrm{mL})$. The solid formed was collected by filtration, washed with ethanol $(20 \mathrm{~mL})$, dried, and crystallized from dioxane as an pale yellow powder in $85 \%$ yield; m.p. $260^{\circ} \mathrm{C}-261^{\circ} \mathrm{C}$, IR ( $\left.\mathrm{cm}^{-1}, v\right): 3435$ (brs, NH's), 1717 , $1680(2 \mathrm{C}=\mathrm{O}) ;{ }^{1} \mathrm{H}$ NMR (DMSO- $\left.d_{6}\right) \mathrm{ppm}: \delta 1.00(\mathrm{~s}, 3 \mathrm{H}$, $\left.\mathrm{CH}_{3}\right), 1.05\left(\mathrm{~s}, 3 \mathrm{H}, \mathrm{CH}_{3}\right), 2.13\left(\mathrm{~m}, 4 \mathrm{H}, 2 \mathrm{CH}_{2}\right), 2.45(\mathrm{~s}, 3 \mathrm{H}$, $\mathrm{CH}_{3}$ ), 9.40 ( br, $\mathrm{NH}$ ); ${ }^{13} \mathrm{C}$ NMR (DMSO- $\left.d_{6}\right)$ ppm: $\delta$, 187.9 (CO), 164.6 (CO amide), 163.8 (C-triazoloe), 160.4 (C-2, pyrimidine), 141.5, 139.1, 124.5, 114.7 (thiophene carbons), 52.21, $44.07\left(2 \mathrm{CH}_{2}\right), 40.61\left(\mathrm{C}\left(\mathrm{CH}_{3}\right)_{2}\right)$, 29.42, 28.05, $27.89\left(3 \mathrm{CH}_{3}\right)$; Its $\mathrm{MS}(\mathrm{m} / \mathrm{z}), 317\left(\mathrm{M}^{+}, 41 \%\right)$; $\mathrm{C}_{14} \mathrm{H}_{15} \mathrm{~N}_{5} \mathrm{O}_{2} \mathrm{~S}$ (317.3); Requires (Found): C, 52.98 (52.96); H, 4.76 (4.74); N, 22.06 (22.03).

Synthesis of 1,4-diamino-8,8-dimethyl-8,9-dihydro[1]benzothieno[3,2-e][1,2,4]triazolo[4,3-a]pyrimidine5,6-(4H,7H)-dione (8c). A mixture of 7 (10 m mol) and potassium thiocyanate $(15 \mathrm{~m} \mathrm{~mol})$ was heated under reflux in glacial acetic acid $(30 \mathrm{~mL})$ for $8 \mathrm{~h}$. The reaction mixture was allowed to cool to room temperature and was poured into water. The precipitate formed was collected by filtration, dried and crystallized from ethanol/dioxane $(2: 1)$ as a yellow powder in $80 \%$ yield. m.p. $210^{\circ} \mathrm{C}-212^{\circ} \mathrm{C}, \mathrm{IR}\left(\mathrm{cm}^{-1}, v\right): 3450$ (brs, NH's), 1721, $1665(2 \mathrm{C}=\mathrm{O}) ;{ }^{1} \mathrm{H} \mathrm{NMR}\left(\mathrm{DMSO}-d_{6}\right): \delta 0.99\left(\mathrm{~s}, 3 \mathrm{H}, \mathrm{CH}_{3}\right)$, $1.02\left(\mathrm{~s}, 3 \mathrm{H}, \mathrm{CH}_{3}\right), 2.13\left(\mathrm{~m}, 4 \mathrm{H}, 2 \mathrm{CH}_{2}\right), 9.10,9.80$ ( brs, $2 \mathrm{NH})$; Its $\mathrm{MS}(\mathrm{m} / \mathrm{z}), 318\left(\mathrm{M}^{+}, 32 \%\right) ; \mathrm{C}_{13} \mathrm{H}_{14} \mathrm{~N}_{6} \mathrm{O}_{2} \mathrm{~S}$ (318.3); Requires (Found): C, 49.04 (49.02); H, 4.43 (4.40); N, 26.40 (26.38).

Synthesis of diamino(methyl or pheynel)benzothieno imidazopyrimidine dione $(9 a, b)$. General procedure: A mixture of compound $7(10 \mathrm{~m} \mathrm{~mol})$ and chloroacetone or 2-bromoacetophenone $(10 \mathrm{~m} \mathrm{~mol})$ was heated under reflux for $12 \mathrm{~h}$ in dry xylene $(30 \mathrm{~mL})$. The solid that separated upon cooling was filtered off and crystallized from appropriate solvent to produce $\mathbf{9 a}, \mathbf{b}$.

Synthesis of 3,4-diamino-2,8,8-trimethyl-3a,4,8,9tetrahydro[1]benzothieno[3,2-e]imidazo[1,2-a]pyrimidine-5,6-(3H,7H)-dione (9a). Compound 9a was obtained from compound $7(10 \mathrm{~m} \mathrm{~mol})$ and chloroacetone $(10 \mathrm{~m} \mathrm{~mol})$ as white crystals crystallized from ethanol in $69 \%$ yield; m.p. $249^{\circ} \mathrm{C}-251^{\circ} \mathrm{C}$, IR $\left(\mathrm{cm}^{-1}, v\right): 3440$ (brs, NH's), 1715, $1669(2 \mathrm{C}=\mathrm{O}) ;{ }^{1} \mathrm{H}$ NMR (DMSO- $\left.d_{6}\right): \delta 0.98$ $\left(\mathrm{s}, 3 \mathrm{H}, \mathrm{CH}_{3}\right), 1.01\left(\mathrm{~s}, 3 \mathrm{H}, \mathrm{CH}_{3}\right), 2.10\left(\mathrm{~m}, 4 \mathrm{H}, 2 \mathrm{CH}_{2}\right)$, $2.23\left(\mathrm{~s}, 3 \mathrm{H}, \mathrm{CH}_{3}\right), 5.70$ (s, 1H, imidazol proton), 6.31 (s,1H, pyrimidine proton), 9.60, 9.85 (brs, 2NH); ${ }^{13} \mathrm{C}$ NMR (DMSO- $\left.d_{6}\right): \delta, 188.3$ (CO), 163.5 (CO amide), 158.4, 154.6 (2C-imidazol), 140.9, 138.7, 123.6, 113.9 (thiophene carbons), 65.7 (C-2, pyrimidine), 53.19, 43.57

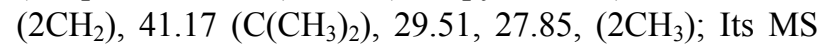
$(\mathrm{m} / \mathrm{z}), 332\left(\mathrm{M}^{+}, 38 \%\right) ; \mathrm{C}_{15} \mathrm{H}_{19} \mathrm{~N}_{5} \mathrm{O}_{2} \mathrm{~S}$ (333.4); Requires (Found): C, 54.02 (54.00); H, 5.74 (5.72); N, 21.01 (21.00).

Synthesis of 3,4-diamino-8,8-dimethyl-2-phenyl-3a, 4,8,9-tetrahydro[1]benzothieno[3,2-e]imidazo[1,2-a] pyrimidine-5,6-(3H,7H)-dione (9b). Compound $9 \mathrm{~b}$ was obtained from compound $7(10 \mathrm{~m} \mathrm{~mol})$ and 2-bromoacetophenone $(10 \mathrm{~m} \mathrm{~mol})$ as a yellow powder crystallized from ethanol in $75 \%$ yield; m.p. $262^{\circ} \mathrm{C}-264^{\circ} \mathrm{C}$, IR $\left(\mathrm{cm}^{-1}, v\right): 3425$ (brs, NH's), 1718, $1675(2 \mathrm{C}=\mathrm{O}) ;{ }^{1} \mathrm{H}$ NMR (DMSO- $\left.d_{6}\right): \delta 1.02\left(\mathrm{~s}, 3 \mathrm{H}, \mathrm{CH}_{3}\right), 1.08\left(\mathrm{~s}, 3 \mathrm{H}, \mathrm{CH}_{3}\right)$, $2.11\left(\mathrm{~m}, 4 \mathrm{H}, 2 \mathrm{CH}_{2}\right), 5.46$ (s, $1 \mathrm{H}$, imidazol proton), 6.09 (s,1H, pyrimidine proton), 7.09 ( $\mathrm{m}, 2 \mathrm{H}$, phenyl proton), 7.34 (m, 3H, phenyl proton), 8.95, 9.60 ( brs, 2NH's); Its MS (m/z), $395\left(\mathrm{M}^{+}, 42 \%\right) ; \mathrm{C}_{20} \mathrm{H}_{21} \mathrm{~N}_{5} \mathrm{O}_{2} \mathrm{~S}$ (395.4); Requires (Found): C, 60.73 (60.70); H, 5.35 (5.33); N, 17.71 (17.69).

Synthesis of 3-amino pyrazolyl benzothieno[2,3-d] pyrimidine-4,5-dione (10a,b). General procedure: A mixture of compound $7(10 \mathrm{~m} \mathrm{~mol})$ and $\beta$-diketone $(10 \mathrm{~m}$ $\mathrm{mol})$ in absolute ethanol $(30 \mathrm{~mL})$ was stirred under reflux for $5 \mathrm{~h}$. The reaction mixture was allowed to cool to $0^{\circ} \mathrm{C}$ for $3 \mathrm{~h}$, the precipitate was filtered off, dried and crystal- 
lized from an appropriate solvent to produce 10a,b.

Synthesis of 3-amino-2-(3,5-dimethyl-1H-pyrazol-1yl)-7,7-dimethyl-3,6,7,8-tetrahydro[1]benzothieno[2,3d]pyrimidine-4,5-dione (10a). Was obtained from 7 $(0.01 \mathrm{~mol})$ with pentan-2,4-dione $(0.01 \mathrm{~mol})$ as gray powder from dioxane in $63 \%$ yield; m.p. $200^{\circ} \mathrm{C}-202^{\circ} \mathrm{C}$, IR $\left(\mathrm{cm}^{-1}\right.$, v) $3410(\mathrm{br}, \mathrm{NH}), 1723,1667(2 \mathrm{C}=\mathrm{O}) ;{ }^{1} \mathrm{H}$ NMR (DMSO- $\left.d_{6}\right): \delta 0.99\left(\mathrm{~s}, 3 \mathrm{H}, \mathrm{CH}_{3}\right), 1.03\left(\mathrm{~s}, 3 \mathrm{H}, \mathrm{CH}_{3}\right)$, $2.15\left(\mathrm{~m}, 4 \mathrm{H}, 2 \mathrm{CH}_{2}\right), 2.26\left(\mathrm{~s}, 3 \mathrm{H}, \mathrm{CH}_{3}\right), 2.85\left(\mathrm{~s}, 3 \mathrm{H}, \mathrm{CH}_{3}\right)$, 6.03 (s, $1 \mathrm{H}$, pyrazolyl proton), 9.60 ( brs, $\mathrm{NH}) ;{ }^{13} \mathrm{C} \mathrm{NMR}$ (DMSO- $d_{6}$ ): $\delta, 187.9$ (CO), 164.6 (CO amide), 159.8 (C-2, pyrimidine), 148.1, 144.2, 143.86 (pyrazol proton), 142.3, 139.5, 124.2, 114.6 (thiophene carbons), 53.08,

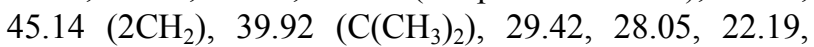
$22.03\left(4 \mathrm{CH}_{3}\right)$; Its MS (m/z), $357\left(\mathrm{M}^{+}, 34 \%\right) ; \mathrm{C}_{17} \mathrm{H}_{19} \mathrm{~N}_{5} \mathrm{O}_{2} \mathrm{~S}$ (357.4); Requires (Found): C, 57.12 (57.10); H, 5.35 (5.33); N, 19.59 (19.58).

Synthesis of 3-amino-2-(4-chloro-3,5-dimethyl-1Hpyrazol-1-yl)-7,7-dimethyl-3,6,7,8-tetrahydro[1]benzo thieno[2,3-d]pyrimidine-4,5-dione (10b). Was obtained from 7 with 3-chloropentan-2,4-dione $(10 \mathrm{~m} \mathrm{~mol})$ as a light yellow powder crystallized from ethanol in $77 \%$ yield; m.p. $210^{\circ} \mathrm{C}-212^{\circ} \mathrm{C}$, IR $\left(\mathrm{cm}^{-1}, v\right): 3400(\mathrm{br}, \mathrm{NH})$, $1715,1668(2 \mathrm{C}=\mathrm{O}) ;{ }^{1} \mathrm{H}$ NMR (DMSO- $\left.d_{6}\right): \delta 0.96(\mathrm{~s}, 3 \mathrm{H}$, $\left.\mathrm{CH}_{3}\right), 1.01\left(\mathrm{~s}, 3 \mathrm{H}, \mathrm{CH}_{3}\right), 2.12\left(\mathrm{~m}, 4 \mathrm{H}, 2 \mathrm{CH}_{2}\right), 2.23(\mathrm{~s}, 3 \mathrm{H}$, $\left.\mathrm{CH}_{3}\right), 2.78$ (s, 3H, $\left.\mathrm{CH}_{3}\right), 9.48$ (brs, NH); Its $\mathrm{MS}(\mathrm{m} / \mathrm{z})$, $391\left(\mathrm{M}^{+}, 25 \%\right),\left(\mathrm{M}^{+}+1,19 \%\right) ; \mathrm{C}_{17} \mathrm{H}_{18} \mathrm{Cl} \mathrm{N}_{5} \mathrm{O}_{2} \mathrm{~S}(391.8)$; Requires (Found): C, 52.10 (52.09); H, 4.62 (4.60); N, 17.87 (17.86).

Synthesis of 3-amino(morpholinyl/methylpiperazin/ and or piperazinyl)-benzothienopyrimidine-4,5-dione (11a-c). General procedure: A mixture of compound 6 (10 m mol) fused with morphline/methylpiprazine/and or piprazine $(15 \mathrm{~m} \mathrm{~mol})$ in sand bath at $180^{\circ} \mathrm{C}$ for $3 \mathrm{~h}$. The reaction mixture was allowed to cool to room temp., and then add $20 \mathrm{~mL}$ of ethanol the precipitate was filtered off, dried and crystallized from an appropriate solvent to produce 11a-c.

Synthesis of 3-amino-7,7-dimethyl-2-morpholin-4yl-3,6,7,8-tetrahydro[1]benzothieno[2,3-d]pyrimidine4,5-dione (11a). It was obtained from 6 with morpholine $(15 \mathrm{~m}$ mol) as a brown powder crystallized from dioxane in $74 \%$ yield; m.p. $237^{\circ} \mathrm{C}-240^{\circ} \mathrm{C}$, IR $\left(\mathrm{cm}^{-1}\right.$, v): $3410(\mathrm{br}$, $\mathrm{NH}), 1720,1675(2 \mathrm{C}=\mathrm{O}) ;{ }^{1} \mathrm{H}$ NMR (DMSO- $\left.d_{6}\right): \delta 0.98$ (s, 3H, $\left.\mathrm{CH}_{3}\right), 1.03\left(\mathrm{~s}, 3 \mathrm{H}, \mathrm{CH}_{3}\right), 2.10\left(\mathrm{~m}, 4 \mathrm{H}, 2 \mathrm{CH}_{2}\right)$, 3.24 (t, 4H, morpholinyl $2 \mathrm{NCH}_{2}, J=5.0 \mathrm{~Hz}$ ), 3.67 (t, $4 \mathrm{H}$, morpholinyl $2 \mathrm{OCH}_{2}, J=4.98 \mathrm{~Hz}$ ), 9.45 (brs, $\left.\mathrm{NH}\right) ;{ }^{13} \mathrm{C}$ NMR (DMSO- $\left.d_{6}\right): \delta, 188.7$ (CO), 166.3 (CO amide), 163.6 (C-2, pyrimidine), 141.4, 139.7, 124.5, 114.3 (thiophene carbons), 66.54, $47.09\left(4 \mathrm{C}, \mathrm{O}\left(\mathrm{CH}_{2}\right)_{2}, \mathrm{~N}\left(\mathrm{CH}_{2}\right)_{2}\right)$ $51.35,43.65\left(2 \mathrm{CH}_{2}\right), 40.19\left(\mathrm{C}_{\left.\left(\mathrm{CH}_{3}\right)_{2}\right), 29.67,28.03}\right.$ $\left(2 \mathrm{CH}_{3}\right)$; Its $\mathrm{MS}(\mathrm{m} / \mathrm{z}), 348\left(\mathrm{M}^{+}, 34 \%\right) ; \mathrm{C}_{16} \mathrm{H}_{20} \mathrm{~N}_{4} \mathrm{O}_{3} \mathrm{~S}$ (348.4); Requires (Found): C, 55.15 (55.12); H, 5.78 (5.74); N, 16.08 (16.05).
Synthesis of 3-amino-7,7-dimethyl-2-(4-methyl piperazin-1-yl)-3,6,7,8-tetrahydro[1]benzo-thieno[2,3d]pyrimidine-4,5-dione (11b). It was obtained from 6 with methylpiprazine $(15 \mathrm{~m} \mathrm{~mol})$ as a yellow powder crystallized from DMF in $68 \%$ yield; m.p. $232^{\circ} \mathrm{C}-234^{\circ} \mathrm{C}$, IR $\left(\mathrm{cm}^{-1}, v\right): 3390(\mathrm{br}, \mathrm{NH}), 1715,1669(2 \mathrm{C}=\mathrm{O}) ;{ }^{1} \mathrm{H}$ NMR (DMSO- $\left.d_{6}\right): \delta 0.96\left(\mathrm{~s}, 3 \mathrm{H}, \mathrm{CH}_{3}\right), 1.01\left(\mathrm{~s}, 3 \mathrm{H}, \mathrm{CH}_{3}\right)$, $2.09\left(\mathrm{~m}, 4 \mathrm{H}, 2 \mathrm{CH}_{2}\right), 2.30\left(\mathrm{~s}, 3 \mathrm{H}\right.$, piperazinyl $\left.\mathrm{NCH}_{3}\right), 2.53$ (brs, $4 \mathrm{H}$, piperazinyl $2 \mathrm{NCH}_{2}$ ), 3.36 (brs, $4 \mathrm{H}$, piperazinyl $2 \mathrm{NCH}_{2}$ ), 9.76 (brs, $\mathrm{NH}$ ); ${ }^{13} \mathrm{C}$ NMR (DMSO- $d_{6}$ ): $\delta, 189.7$ (CO), 164.9 (CO amide), 161.4 (C-2, pyrimidine), 142.3, 140.4, 124.6, 113.9 (thiophene carbons), 56.47, 46.18 $\left(4 \mathrm{C}, \mathrm{N}\left(\mathrm{CH}_{2}\right)_{2}, \mathrm{~N}\left(\mathrm{CH}_{2}\right)_{2}\right)$ 50.85, $42.93\left(2 \mathrm{CH}_{2}\right), 41.19$ $\left(\mathrm{C}\left(\mathrm{CH}_{3}\right)_{2}\right), 30.02,29.01\left(2 \mathrm{CH}_{3}\right)$; Its $\mathrm{MS}(\mathrm{m} / \mathrm{z}), 361\left(\mathrm{M}^{+}\right.$, 29\%); $\mathrm{C}_{17} \mathrm{H}_{23} \mathrm{~N}_{5} \mathrm{O}_{2} \mathrm{~S}$ (361.4); Requires (Found): C, 56.48 (56.45); H, 6.41 (6.39); N, 19.37 (19.35).

Synthesis of 3-amino-7,7-dimethyl-2-piperazin-1yl)-3,6,7,8-tetraahydro[1]benzothieno[2,3-d]pyrimidine-4,5-dione (11c). It was obtained from 6 with piprazine $(15 \mathrm{~m} \mathrm{~mol})$ as a pale yellow powder crystallized from DMF in $65 \%$ yield; m.p. $250^{\circ} \mathrm{C}-252^{\circ} \mathrm{C}$, IR $\left(\mathrm{cm}^{-1}\right.$, v): 3400 (br, $\mathrm{NH}), 1713,1667 \quad(2 \mathrm{C}=\mathrm{O}) ;{ }^{1} \mathrm{H}$ NMR (DMSO- $\left.d_{6}\right): \delta 1.01\left(\mathrm{~s}, 3 \mathrm{H}, \mathrm{CH}_{3}\right), 1.04\left(\mathrm{~s}, 3 \mathrm{H}, \mathrm{CH}_{3}\right), 2.11$ (m, $4 \mathrm{H}, 2 \mathrm{CH}_{2}$ ), 2.48 (brs, $4 \mathrm{H}$, piperazinyl $2 \mathrm{NCH}_{2}$ ), 3.29 (brs, $4 \mathrm{H}$, piperazinyl $2 \mathrm{NCH}_{2}$ ), 9.76, 10.15 (brs, NH's); Its MS (m/z), $347\left(\mathrm{M}^{+}, 38 \%\right) ; \mathrm{C}_{16} \mathrm{H}_{21} \mathrm{~N}_{5} \mathrm{O}_{2} \mathrm{~S}$ (347.4); Requires (Found): C, $55.30(55.28) ; \mathrm{H}, 6.09(6.06) ; \mathrm{N}$, 20.15 (20.13).

\subsection{Biological Screening}

\subsubsection{Materials and Methods}

Animals-adult rats of both sexes weighing $150-200 \mathrm{~g}$ and adult mice weighing $20-25 \mathrm{~g}$ were used in the experiments. Animals were housed under standardized conditions for light and temperature and received standard rat chow and tap water and libitum. Animals were randomly assigned to different experimental groups, each kept in a separate cage. All animal procedures were performed after approval from the Ethics committee of the National Research Center and in accordance with the recommendations for the proper care and use of laboratory animals (NIH publication No. 85-23, revised 1985).

\subsubsection{Antiinflammatory Testing}

The carrageenan rat paw edema model of inflammation was used to evaluate the anti-inflammatory properties of the tested compounds. Rats were randomly assigned to the treatment groups and sterile carrageenan lambda (100 ul of a $1 \%$ solution in saline) was injected sub-planter into right hind paw of the rat. Carrageenan caused visible redness and pronounced swelling that was well developed by $4 \mathrm{~h}$ and persisted for more than $48 \mathrm{~h}$. Right hind paw was measured with a planimeter $[24,25]$ before, and at 1,2, 3 and $4 \mathrm{~h}$ after carrageenan injection. All the 
tested compounds were dissolved in DMSO then injected i.p. $(9 \mathrm{mg} / \mathrm{kg}$ b wt). The control animals were injected (i.p.) with appropriate volume of DMSO. The standard drug was indomethacin $(10 \mathrm{mg} / \mathrm{kg} \mathrm{b} \mathrm{wt})$. Different compounds or indomethacin were given $1 \mathrm{hr}$ before carrageenan injection.

\subsubsection{Analgesic Testing}

The hot-plate test was performed on mice by using an electronically controlled hot-plate (Ugo Basile, Italy) heated to $52^{\circ} \mathrm{C}$, for possible centrally mediated analgesic effect of the drugs. Fourteen groups of rats were given vehicle and/or the different compounds and the last group received tramadol $(20 \mathrm{mg} / \mathrm{kg} \mathrm{b} \mathrm{wt}) 60 \mathrm{~min}$ prior to testing. Latency to lick a hind paw or jumping [26] was recorded sequentially before and at $1,2 \mathrm{~h}$ post treatment.

\subsubsection{Ulcerogenic Effects}

Groups of five male Wistar rats with a weight between 150 and $175 \mathrm{~g}$ are used. They are starved $48 \mathrm{~h}$ prior to drug administration. The test compounds are administered orally in $10 \mathrm{~mL} / \mathrm{kg}$ as aqueous suspension. Doses which are highly active in the activity $(9 \mathrm{mg} / \mathrm{kg})$ are chosen and used. The animals are sacrificed after $7 \mathrm{~h}$. Stomachs are removed and placed on saline soaked filter paper until inspection. A longitudinal incision along the greater curvature is made with fine scissor. The stomach is inverted over the index finger and the presence or the absence of gastric irritation is determined. The presence of a single or multiple lesions (erosion, ulcer or perforation) is considered to be positive [23]. The number of ulcers and the occurrence of hyperemia is noted (determine ulcer index).

\section{Acknowledgements}

The authors are thankful to the Al-Imam Mohammad Ibn Saud Islamic University (IMSIU), Faculty of Science, for providing laboratory facilities, Micro-analytical Centre, and the Pharmacological Unit National Research Centre, for microanalyses and pharmacological screening of the compounds.

\section{REFERENCES}

[1] A. J. Folkes, K. Ahmadi, W. K. Alderton, S. Alix, S. J. Baker, G. Box, I. S. Chuckowree, P. A. Clarke, P. Depledge, S. A. Eccles, L. S. Friedman, A. Hayes, T. C. Hancox, A. Kugendradas, L. Lensun, P. Moore, A. G. Olivero, J. Pang, S. Patel, G. H. Pergl-Wilson, F. I. Raynaud, A. Robson, N. Saghir, L. Salphati, S. Sohal, M. H. Ultsch, M. Valenti, H. J. A. Wallweber, N. C. Wan, C. Wiesmann, P. Workman, A. Zhyvoloup, M. J. Zvelebil and S. J. Shuttleworth, "The Identification of 2-(1H-Indazol-4-yl)-6-(4methanesulfonylpiperazin-1-yl-methyl)-4-morpholin-4yl-thie-no[3,2- $d$ ]pyrimidine (GDC-0941) as a Potent, Se- lective, Orally Bioavailable Inhibitor of Class I PI3 Kinase for the Treatment of Cancer," Journal Medicinal Chemistry, Vol. 51, No. 18, 2008, pp. 5522-5532.

[2] L. D. Jennings, S. L. Kincaid, Y. D. Wang, G. Krishnamurthy, C. F. Beyer, J. P. McGinnis, M. Miranda, C. M. Discafani and S. K. Rabindran, "Parallel Synthesis and Biological Evaluation of 5,6,7,8-Tetrahydrobenzothieno [2,3-d]pyrimidin-4(3H)-one Cytotoxic Agents Selective for p21-Deficient Cells," Bioorganic \& Medicinal Chemistry Letter, Vol. 15, No. 21, 2005, pp. 4731-4735.

[3] Y. D. Wang, S. Johnson, D. Powell, J. P. McGinnis, M. Miranda and S. K. Rabindran, "Inhibition of Tumor Cell Proliferation by Thieno[2,3- $d$ ]pyrimidin-4(1H)-one-based Analogs," Bioorganic \& Medicinal Chemistry Letter, Vol. 15, No. 16, 2005, pp. 3763-3766.

[4] T. Horiuchi, J. Chiba, K. Uoto and T. Soga, "Discovery of Novel Thieno[2,3-d]pyrimidin-4-yl Hydrazone-Based Inhibitors of Cyclin D1-CDK4: Synthesis, Biological Evaluation, and Structure-Activity Relationships," Bioorganic \& Medicinal Chemistry Letter, Vol. 19, No. 2, 2009, pp. 305-308.

[5] A. Angell, C. McGuigan, L. G. Sevillano, R. Snoeck, G. Andrei, E. De Clercq and J. Balzarini "Bicyclic AntiVZV Nucleosides," Bioorganic \& Medicinal Chemistry Letter, Vol. 14, No. 10, 2004, pp. 2397-2399.

doi:10.1016/j.bmcl.2004.03.029

[6] A. Brancale, C. Mcguigan, B. Algain, S. Pascal, R. Benhida, J. L. Fourrey, G. Andrei, R. Snoeck, E. De Clercq and J. Balzarini, "Bicyclic Anti-VZV Nucleosides: Thieno Analogues Retain Full Antiviral Activity," Bioorganic \& Medicinal Chemistry Letter, Vol. 11, No. 18, 2001, pp. 2507-2510.

[7] M. H. Bhuiyan. K. M. Rahman, K. Hossain, A. Rahim, I. Hossain and M. Abu Naser, "Synthesis and Antimicrobial Evaluation of Some New Thienopyrimidine Derivatives," Acta pharmaceutica, Vol. 56, No. 4, 2006, pp. 441-450.

[8] R. V. Chambhare, B. G. Khadse, A. S. Bobde and R. H. Bahekar, "Synthesis and Preliminary Evaluation of Some $N$-[5-(2-Furanyl)-2-methyl-4-oxo-4H-thieno[2,3- $d$ ]pyrimi din-3-yl]carboxamide and 3-Substituted-5-(2-furanyl)-2methyl-3H-thieno[2,3- $d$ ]pyrimidin-4-ones as Antimicrobial Agents," European Journal of Medicinal Chemistry, Vol. 38, No. 1, 2003, pp. 89-100.

[9] S. Yousieff and B. E. Bayoumy, "1,3,4-Thiadiazolylthieno Pyrimidines and 1,3,4-Oxadiazolyl Thienopyrimidines for Antibacterial Activity," Journal of Pharmaceutical Sciences, Vol. 31, No. 1-3, 1990, p. 67.

[10] V. Alagarsamy, S. Vijayakumar and V. R. Solomon, "Synthesis of 2-Mercapto-3-substituted-5,6-dimethylthieno[2,3- $d]$ Pyrimidin-4(3H)-ones as New Analgesic, AntiInflammatory Agents," Biomed Pharmacother, Vol. 61, No. 5, 2007, pp. 285-291. doi:10.1016/j.biopha.2007.02.008

[11] V. Alagarsamy, S. Meena, K. V. Ramseshu, V. R. Solomon, K. Thirumurugan, K. Dhanabal and M. Murugan, "Synthesis, Analgesic, Anti-Inflammatory, Ulcerogenic Index and Antibacterial Activities of Novel 2-Methylthio3-substituted-5,6,7,8-tetrahydrobenzo( $b$ )thieno[2,3- $d]$ pyri midin-4(3H)-ones," European Journal of Medicinal Che- 
mistry, Vol. 41, No. 11, 2006, pp. 1293-1300.

[12] I. O. Donkor, L. I. Hui and S. F. Queener, "Synthesis and DHFR Inhibitory Activity of a Series of 6-Substituted2,4-diaminothieno[2,3-d]pyrimidines," European Journal of Medicinal Chemistry, Vol. 38, No. 6, 2003, pp. 605611.

[13] H. N. Hafez and A. B. A. El-Gazzar, "Design and Synthesis of 3-Pyrazolyl-thiophene, Thieno[2,3- $d$ ]pyrimidines as New Bioactive and Pharmacological Activities," Bioorganic \& Medicinal Chemistry Letter, Vol. 18, No. 19, 2008, pp. 5222-5227.

[14] A. B. A. El-Gazzar, H. A. R. Hossein and H. N. Hafez, "Synthesis and Biological Evaluation of Thieno[2,3d]pyrimidine Derivatives as Anti-inflammatory, Analgesic and Ulcerogenic Activity," Acta Pharmaceutica, Vol. 57, No. 4, 2007, pp. 395-411. doi:10.2478/v10007-007-0032-6

[15] M. Santagati, M. Modica, A. Santagati, F. Russo and S. Spampinato, "Synthesis of Aminothienopyrimidine and Thienotriazolopyrimidine Derivatives as Potential Anticonvulsant Agents," Die Pharmazie, Vol. 51, No. 1, 1996, pp. 7-11.

[16] M. R. Prasad, R. A. Raghuram, R. P. Shanthan, R. K. Subramanian, S. Meena and K. Madhavi, "Synthesis and Adenosine Receptor Binding Studies of Some Novel Triazolothienopyrimidines," European Journal of Medicinal Chemistry, Vol. 43, No. 3, 2008, pp. 614-620.

[17] L. P. Melissal, C. G. Wayne, E. Tara, A. N. Jason, L. Patricia, C. J. José, D. Fernando and J. T. Robert, "Design of Novel $N$-(2,4-Dioxo-1,2,3,4-tetrahydro-thieno[3,2-d] pyrimidin-7-yl)-guanidines as Thymidine Phosphorylase Inhibitors, and Flexible Docking to a Homology Model," Bioorganic \& Medicinal Chemistry Letter, Vol. 13, No. 1, 2003, pp. 107-110.

[18] H. N. Hafez, H. A. Hussein and A. B. A. El-Gazzar, "Synthesis of Substituted Thieno[2,3- $d$ ] pyrimidine-2,4dithiones and Their $S$-Glycoside Analogues as Potential Antiviral and Antibacterial Agents," European Journal of Medicinal Chemistry, Vol. 45, No. 9, 2010, pp. 4026-
4034.

[19] C. J. Shishoo, V. S. Shirsath, I. S. Rathod, M. J. Patil and S. S. Bhargava, "Design Synthesis and Antihistaminic (H1) Activity of Some Condensed 2-(Substituted)arylaminoethylpyrimidine-4-(3H)-ones," Arzneim Forsch, Vol. 51, No. 3, 2001, pp. 221-231.

[20] G. A. Winter, E. A. Rislfy and G. W. Nuss, "Carrageenininduced Edema in Hind Paw of Rat as an Assay for Antiinflammatory Drugs," Proceedings of the Society for Experimental Biology and Medicine, Vol. 111, 1962, pp. 544-547.

[21] P. Armitage, "Statistical Methods in Medical Research," 1st Edition, Blackwell Scientific Publ., Oxford, London, 1971.

[22] G. Woolfe and A. D. MacDonald, "The Evaluation of the Analgesic Action of Pethidine Hydrochloride," Journal of Pharmacoogy and Experimental Therapeutics, Vol. 80, No. 3, 1944, pp. 300-307.

[23] A. E. Amr and M. M. Abdulla, "Synthesis and Antiinflammatory Activities of New Cyanopyrane Derivatives Fused with Steroidal Nuclei," Archiv der Pharmazie Chemistry Life Science, Vol. 339, No. 2, 2006. pp. 88-95. doi:10.1002/ardp.200500209

[24] M. G. Obukowics, D. J. Walseh, W. J. Salsgiver, C. L. Martin-Berger, K. S. Chinn, K. L. Duffin, A. Ras and P. Needleman, "Novel, Selective $\Delta 6$ or $\Delta 5$ Fatty Acid Desaturase Inhibitors as Antiinflammatory Agents in Mice," Journal of Pharmacoogy and Experimental Therapeutics, Vol. 287, No. 1, 1998, pp. 157-166.

[25] L. Meng, R. Mohan, B. H. B. Kwok, M. Elofssof, N. Sin and C. M. Crews, "Epoxomicin, a Potent and Selective Proteasome Inhibitor, Exhibits in Vivo Antiinflammatory Activity," Proceedings of the National Academy of Science, USA, Vol. 96, No. 18, 1999, pp. 10403-10408.

[26] M. Eaton, "Common Animal Models for Spasticity and Pain," Journal of Rehabilitation Research and Development, Vol. 40, No. 4, 2003, pp. 41-54. doi:10.1682/JRRD.2003.08.0041 